\title{
The genetic epidemiology of multiple sclerosis
}

\author{
Alastair Compston \\ University of Cambridge Neurology Unit, Addenbrooke's Hospital, Hills Road, Cambridge CB2 2QQ, UK \\ (alastair.compston@medschl.cam.ac.uk)
}

\begin{abstract}
Epidemiological studies have implicated an interplay between genetic and environmental factors in the aetiology of multiple sclerosis (MS). There is a familial recurrence rate of approximately $15 \%$. Metaanalysis of the recurrence risk shows that the rate is highest overall for siblings, then parents and children, with lower rates in second- and third-degree relatives. Recurrence is highest for monozygotic twins. Conversely, the frequency in adoptees is similar to the population lifetime risk. The age-adjusted risk for half siblings is also less than for full siblings. Recurrence is higher in the children of conjugal pairs with MS than the offspring of single affecteds. These classical genetic observations suggest that MS is a complex trait in which susceptibility is determined by several genes acting independently or epistatically. Comparisons between co-affected sibling pairs provide no evidence for correlation with age or year at onset and mode of presentation or disability. Thus far, the identification of susceptibility genes has proved elusive but genetic strategies are now in place which should illuminate the problem. The main dividend will be an improved understanding of the pathogenesis. To date, population studies have demonstrated an association between the class II major histocompatibility complex (MHC) alleles DR15 and DQ6 and their corresponding genotypes. An association with DR4, with or without the primary DR15 link, is seen in some Mediterranean populations. Candidate gene approaches have otherwise proved unrewarding. Four groups of investigators have undertaken a systematic search of the genome. In common with most other complex traits, no major susceptibility gene has been identified but regions of interest have been provisionally identified. These genetic analyses are predicated on the assumption that MS is one disease. Genotypic and phenotypic analyses are beginning to question this assumption. A major part of future studies in the genetics of MS will be to resolve the question of disease heterogeneity.
\end{abstract}

Keywords: multiple sclerosis; aetiology; epidemiology; susceptibility

\section{INTRODUCTION}

The need to understand the aetiology and pathogenesis of multiple sclerosis (MS) as the logical basis for treatment has been appreciated ever since the nosological status of the disease was consolidated towards the end of the 19th century. Two aetiological themes emerge from a consideration of the geographical distribution and recurrence risks in pedigrees - the contributions of genes and the environment. For a while, these were seen as alternatives, jostling for priority in the mechanistic formulations of clinical investigators, but present concepts characterize MS as a typical complex trait in which there is an interplay between individual susceptibility and extrinsic triggers.

\section{THE DISTRIBUTION OF MULTIPLE SCLEROSIS}

Working in the modern era, Kurtzke (1975) first suggested that the global distribution of MS could be classified into low-, medium- and high-prevalence bands. The absolute number of cases identified in different parts of the world has since steadily increased (Kurtzke 1993). This can be explained on the basis of improved diagnosis, increased awareness and better symptomatic treatments leading to improved life expectancy, without necessarily the need to invoke a change in incidence - although that cannot altogether be excluded. It seems that many original claims concerning the existence of latitudinal gradients were overstated although the disease does undoubtedly show variations in its distribution over quite small distances. These may be aetiologically informative but the pattern can be explained either by the effect of a putative extrinsic factor, presumably an infectious agent, or on the basis of population genetics.

MS is common in northern Europe, continental north America and Australasia, but not the Orient, Arabian peninsula, Africa, continental South America or India (for a review, see Compston et al. 1998) (figure 1). Within northern Europe, the disease is more frequent in southern Scandinavia, northern Germany, the UK and parts of Italy than in northern Scandinavia, France, Spain and the eastern Mediterranean countries. Within the UK, the areas of highest prevalence are north-east Scotland and the Orkney and Shetland Islands. In Italy, marked differences in prevalence exist between regions and islands that are geographically close but differ in their genetic and cultural histories. In North America, there is a diagonal gradient in frequency with the highest rates in the mid-West and lowest rates in the Mississippi Delta. A latitudinal gradient exists for the White population in Australia with higher rates in the south than north. In summary, MS is common in areas populated by northern Europeans and infrequent in regions where the indigenous peoples are African, Asian and Oriental. 


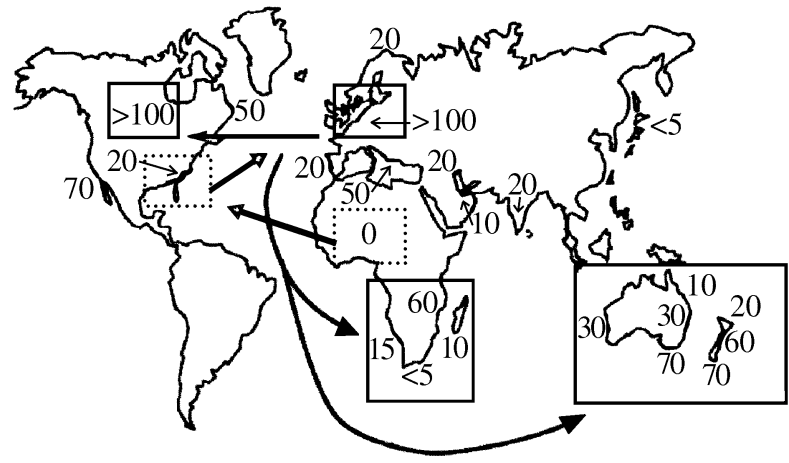

Figure 1. The distribution of MS (prevalence per $10^{5}$ ) with the main informative migration vectors for northern Europeans and Africans: figures for southern Africa are English-speaking Whites migrating as adults (60) or as tchildren (15), Afrikaners (ten) and Cape Coloureds (less than five).

\section{(a) Migration}

As a generalization, studies of geographically stable populations illustrate differences in genetic susceptibility, whereas the effect of migration has been to define MS as an acquired exogenous disorder. These principles are well illustrated by studies of MS in Africans. In southern Africa, the prevalence of MS is relatively high in immigrants from Europe, low in Afrikaners and intermediate in southern African English; occasional cases are seen in the Cape Coloured population which has mixed African and European ancestry and the disease is of legendary rarity in African Blacks. English-speaking Whites moving from northern Europe to southern Africa as adults take with them the high frequency of their country of origin, whereas those migrating below the age of 15 years show the lower rates characteristic of native-born inhabitants of southern Africa (Dean 1967). Looked at in reverse, the prevalence of MS in the UK-born children of West Indian, African and Asian immigrants approximates that seen in similar age groups among Caucasians (Elian \& Dean 1997). The same interplay of race and place emerged from a survey of MS in Israel. The original study in immigrants reported a difference in prevalence between migrants from northern Europe (Ashkenazis) and from Asia and Africa (Sephardis) with an age-atmigration effect in that there were very few affected Ashkenazis in the cohort arriving in Israel before adolescence. Updated estimates also support the hypothesis that, at least for Ashkenazi and Sephardic Jews, racially determined differences in risk of MS are modified by the environment (Kahana et al. 1994).

\section{(b) Epidemics}

More persuasive with respect to the effect of the environment on the development of MS are the apparent epidemics. The first retrospective survey of Iceland showed that each quinquennial rate of incidence from 1900 was lower than between 1945 and 1954, during which age at onset was also conspicuously younger. This suggested a post-war epidemic of MS in Iceland (Kurtzke et al. 1982). The original observations on MS in the Faroe Islands showed fewer cases than expected from comparisons with the neighbouring Orkney and Shetland Islands. Having failed to identify any patient with an estimated date of onset earlier than 1945, a significant increase was claimed from 1943 such that 41 cases had been ascertained by 1986. Kurtzke (1993) concluded that the critical factor determining the Faroe Island's experience of MS was occupation by British troops between 1940 and 1945. The development of MS showed both a temporal and spatial relationship in that villages where individuals who contributed to each of the incidence peaks lived were also those where troops were billeted. However, some are not convinced by these analyses, preferring the view that any change in frequency relates to improved recognition and diagnostic procedures rather than increased incidence (Benedikz et al. 1994).

\section{FACTORS WHICH PREDICT THE CLINICAL COURSE}

Although the individual experience of MS is unpredictable, patterns can be recognized based on multivariate analysis of the clinical features and their course and certain laboratory indices of disease activity. Mechanistically, these are largely unexplained but several provocative life events have been proposed which might provide clues either to the aetiology of MS or the mechanisms of disease activity.

The contribution made by genetic factors to the course of MS can be estimated by comparing age and year at onset in co-affected relatives. Clustering by age at onset suggests a genetic influence and year at onset a strong influence of environmental factors; clustering by neither implies random exposure to an environmental factor. These studies are much prone to confounding, for example concordance for year at onset is influenced by the tendency for an earlier recognition of symptoms in the second individual affected in a pair through heightened awareness of the possibility of MS. It could be argued that comparing year and age at onset is too imprecise for assessing the relative contribution of genes and environmental factors in determining the development of MS in individuals. Bulman et al. (1991) studied 99 sibling pairs and showed a significant intraclass correlation for age at onset in siblings and an even stronger correlation in concordant monozygotic twins. Robertson et al. $(1996 a)$ reported 166 families containing 343 affected individuals providing 177 pairs. After appropriate correction, there was no correlation with age or year at onset. Mode of presentation and disability showed no correlation, suggesting that individuals with familial MS can neither take comfort from nor should they necessarily be concerned about the pattern of disease in an affected relative. There was, however, a correlation with disease course, even after the exclusion of pairs with primary progressive MS, and affected siblings tended to be of the same sex.

\section{(a) Pregnancy}

MS is more common in females than males (ratio females:males of 3:1). Onset of the disease does not cluster around pregnancy and having children does not alter the long-term course of the disease, but there is an increase in the relapse rate during the puerperium. Prospective studies have indicated an approximately threefold higher risk in the three to six months after term than during 
pregnancy and suggested that the attacks may be more severe. Runmarker \& Andersen (1995) studied an inception cohort in Sweden and disposed of the hypothesis that the onset of MS is influenced by pregnancy; in fact, there was a conspicuous absence of onset bouts during pregnancy compared with non-pregnant epochs including the puerperal eight months. Fecundity was reduced in women with MS, presumably by choice, particularly in the context of significant disability and this is the probable explanation for concluding that pregnancy after onset is associated with a lower risk of progression. The most recent large prospective study showed a reduction for each trimester compared to the pre-pregnancy relapse rate (per quartile), with a subsequent increase in the puerperium for 222 completed pregnancies (Confavreux et al. 1998). The preferred interpretation is that these changes result from oscillations in the Th-1 to Th-2 pattern of immune responsiveness which characterize pregnancy and the puerperium.

\section{(b) Virus infection}

There is unequivocal evidence from prospective studies that new episodes of demyelination are more likely to occur following viral exposure (Sibley et al. 1985), particularly upper respiratory (adenovirus) and gastrointestinal infections (Andersen et al. 1993). Nine per cent of presumed infections are followed by relapse and $27 \%$ of new episodes are related to infection. The relative risk for a new episode in the four week period after infection is 1.3. Several studies have attempted to correlate exposure to viral illness in childhood with the subsequent development of MS (for a review, see Granieri \& Casetta 1997). The risk is increased for individuals who develop a variety of exanthematous and other common viral disorders relatively late in childhood. This particularly applies to Epstein-Barr virus infection (Operskalski et al. 1989; Martyn et al. 1993); those reporting infectious mononucleosis before the age of 18 years have an eightfold relative risk of MS.

\section{(c) Trauma}

Several lines of evidence are considered relevant to the debate on whether trauma can trigger clinical manifestations of MS in someone who has the disease process or alter the course in individuals who have already experienced symptoms. Sibley et al. (1991) prospectively studied 170 patients by questionnaire (monthly) and physical examination (three monthly) for eight years. Defining either the three or six month period following each event as 'at risk', only electrical trauma showed an association with new episodes and all other forms of trauma were negatively correlated with both clinical exacerbations and disease progression. Most recently, Siva et al. (1993) identified trauma in the year preceding onset in only three out of 223 incident patients from the Mayo Clinic series; specifically, in prospective studies they failed to show an increased risk after head trauma, limb fracture and cervical or lumbar disc surgery. Several mechanistic hypotheses have been advanced which link trauma to disease activity through a direct effect on permeability of the blood-brain barrier. Against the epidemiological background, further consideration of a causal link between trauma and MS might be consid-

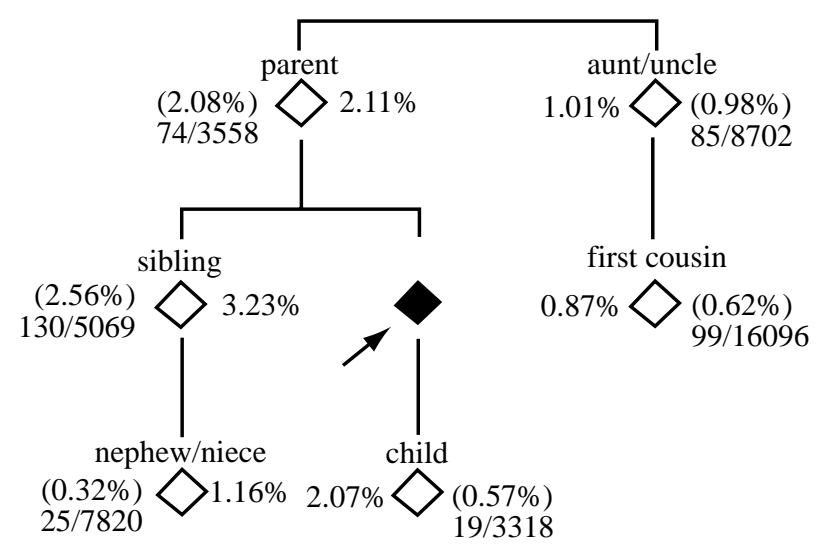

Figure 2. Meta-analysis of the recurrence risks for MS derived from three population-based series showing the crude and age-corrected rates for each category of

relative compared to the proband. See the text for details and references.

ered redundant but, at least in medico-legal circles, the issue is still alive.

\section{THE GENETICS OF MULTIPLE SCLEROSIS}

MS has a familial recurrence rate of around $15 \%$. Pedigree analyses indicate that this mainly results from coinheritance of susceptibility genes rather than shared exposure to a common environmental trigger. Slow but steady progress is being made in identifying and locating the regions encoding genes that confer susceptibility, acting independently or through epistatic effects.

\section{(a) Pedigree analysis}

The most comprehensive studies of recurrence risk are from Canada, the UK and Belgium (Sadovnick et al. 1988; Carton et al. 1997; Robertson et al. 1996b). Metaanalysis of recurrence risk among 44177 relatives of 2163 probands from these population-based series showed that the age-adjusted risk is highest for siblings $(3 \%)$, then parents $(2 \%)$ and children $(2 \%)$, with lower rates in second- and third-degree relatives. Overall, the reduction in risk changes from 3\% (relative risk 9.2) in first-degree relatives to $1 \%$ in second- and third-degree relatives (relative risks 3.4 and 2.9, respectively), compared with a background age-adjusted risk in this population of $0.3 \%$ (figure 2). With some variations in methodology, three recent studies approximated to a population-based series of MS in twins (French Research Group on Multiple Sclerosis 1992; Sadovnick et al. 1993; Mumford et al. 1994). Two showed remarkable consistency in demonstrating a higher clinical concordance rate in monozygotic twins ( $c a$. $25 \%$ ) than dizygotic pairs (ca. 3\%). The French study is exceptional in showing no significant difference between monozygotic and dizygotic twins but this result is within the confidence limits of the other surveys. The relative risk for $\mathrm{MS}$ in the monozygotic twin partner of an affected proband is therefore approximately 190.

Adopted individuals who subsequently develop MS and affecteds who have themselves adopted children provide an unusual but informative resource for studying the relative contribution of genes and the environment in the aetiology of MS. Considering individuals with MS who 
are adopted before the age of one year and those with MS who, through adoption, have non-biological siblings or children, the frequency of MS in non-biological parents, siblings and children is more or less identical to the population prevalence and lifetime risk for northern Europeans and significantly lower than that expected from the study of recurrence risks in the biological relatives of index cases (Ebers et al. 1995). Half siblings offer yet one more variant on the familial MS theme in that they share a proportion of parental genes and divide into those who do and do not share the same environment, at least during the period which is thought critical in the development of MS. The age-adjusted risk for half siblings is significantly lower than for full siblings and there is no difference in risk for half siblings reared together and apart (Sadovnick et al. 1996). Conjugal pairs with MS who have children provide a special opportunity for assessing the contribution made to susceptibility by genetic factors. In the one published study, a much higher recurrence risk (age-adjusted risk 1:5) was observed than for the children of single affecteds (age-adjusted risk 1:50; Robertson et al. 1997). This suggests that the dose of the genetic effect not only increases the absolute risk but also anticipates age at presentation.

\section{(b) Strategies for identifying susceptibility genes}

The advent of laboratory methods for distinguishing short sections of DNA and performing accurate mapping of the many available microsatellites and single nucleotide polymorphisms across the genome, the availability of extended pedigrees in which affected status can reliably be determined and the deployment of appropriate statistical methods for assessing genome-wide significance have made it possible to tackle the genetics of complex traits. Unlike monogenic disorders, the responsible genes are not mutations which code for aberrant gene products but normal polymorphisms. They are multiple and each may exert a very small contributory effect on some as yet undefined structure or physiological function.

Originally, the search for genes determining susceptibility to complex diseases was organized by comparing the frequency of polymorphisms at a given genetic locus in groups of unrelated individuals with and without the trait. These population-based association studies were limited by the number of polymorphic systems which could be explored, the paucity of the hypotheses which directed the search towards a particular region of interest and the confounding effects of inappropriate choice of controls. However, an important principle to emerge was that (to a varying extent) alleles encoded at neighbouring loci tend to co-segregate. It seems that many adjacent markers are not yet randomly distributed by genetic recombinations either because the number of generations over which mitoses have occurred is still too few to establish genetic equilibrium or because there is evolutionary pressure to maintain these linkage disequilibria. From the practical point of view, this means that association studies can be informative even when the marker is up to $0.5 \mathrm{cM}$ distant from the disease susceptibility locus. Hence, for a given effort, the chance of identifying a disease association is increased by linkage disequilibrium.

A change in the strategy for studying the genetics of complex traits became routine over the last decade with the reapplication of previously described family-based methods (Risch 1990). The biased inheritance of genomic regions encoding susceptibility genes for MS in affected family pairs in which ancestral haplotypes had been identified through typing both parents is enshrined both in affected family pair linkage analysis and transmission disequilibrium testing (TDT). In these settings, each is a test for linkage but transmission disequilibrium can also be used to test for association (figure 3).

Family-based linkage methods have been applied most energetically to sibling pairs. The probabilities that, for a given locus, each affected sibling will share neither, one or both parental alleles are $0.25,0.50$ and 0.25 , respectively. Linkage is present if, in a sufficient sample size, that distribution is distorted in favour of one or two sharing. Conceptually, it was then a short step to move from linkage analysis of defined loci to a systematic search of the genome without prejudice as to how many and where the susceptibility genes might be located. Given the number of tests being carried out, some work was needed on an estimation of statistical significance. Assessment of the probability that any one genetic locus might encode a susceptibility gene then advanced to methods for creating multipoint maps, in which information from adjacent loci is used to maximize the statistical statement. The evidence relating to a given locus can be expressed as the logarithm of the odds (lod) for a gene being encoded at that site (the maximum lod score) or, conversely, as the probability that such a gene is not there encoded. Since linkage analysis is very unlikely to reveal the precise identity of a susceptibility gene in one step but merely narrows the search to a region of interest which may contain very many positional candidates, these exclusion maps are useful as a means of discarding unrewarding sections of the genome for further study. The difficulty is that exclusion maps have to be set for an arbitrary level of biological importance and mode of inheritance. Each may be difficult to establish. Genes of major significance can be excluded much more easily than those which contribute rather little to disease susceptibility. It remains a problem for the clinical scientist to judge just how much effort is worth investing in questions for which the answers may only provide trivial details on the nature of susceptibility.

Because it traces the movement of allelic markers present on each chromosome across one generation (mitosis), linkage analysis provides information concerning relatively large parts of the genome. It is efficient for detecting genes exerting major effects but the power of linkage analysis is approximately inversely related to the square root of the contribution made to susceptibility and it is therefore not efficient for genes exerting minor biological effects. Based on existing results, calculations on the sample size of affected relative pairs needed to resolve the genetics of MS are distinctly gloomy.

For this reason, a further switch in strategy has occurred in the last few years towards family-based association studies. In its simplest form, TDT uses trios consisting of single affecteds with both parents (who usually are but do not need to be unaffected). The aim is to show that, in a sufficient sample size, alleles encoded at a particular locus are transmitted to the affected individual more or less often than expected by chance. Each 


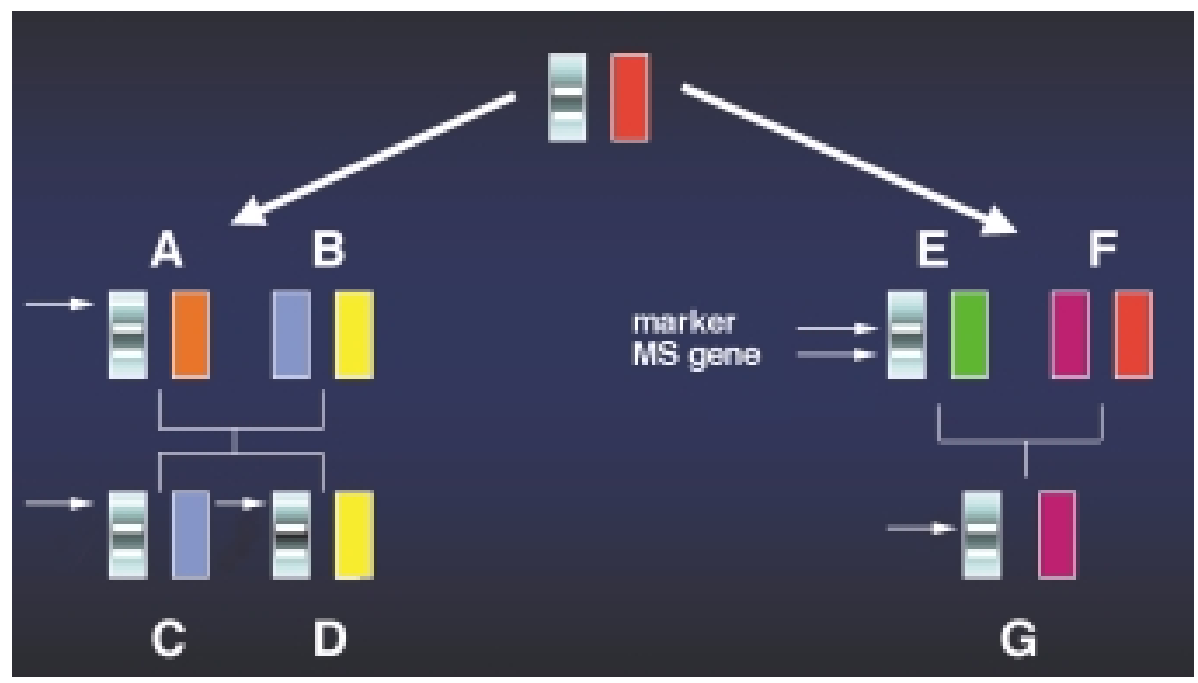

Figure 3. The principles of identifying regions of interest for susceptibility genes in MS using affected family pairs or transmission disequilibrium in trios. The genomic region bounded by the putative MS susceptibility gene and its marker is transmitted in linkage disequilibrium from individual E to G. An additional marker, outside the region of linkage disequilibrium, shown as an additional horizontal band in individual $\mathrm{A}$, is transmitted to the affected sibling pair $\mathrm{C}$ and $\mathrm{D}$. The case-control study compares $\mathrm{C}+\mathrm{G}+\ldots$ with $\mathrm{X}+\mathrm{Y}+\ldots$ Sibling pair linkage uses $\mathrm{C}+\mathrm{D}$ with $\mathrm{A}+\mathrm{B}$. Transmission disequilibrium uses $\mathrm{G}$ or $\mathrm{D}$ with $\mathrm{A}+\mathrm{B}$ or $\mathrm{G}$ with $\mathrm{E}+\mathrm{F}$. See the text for details.

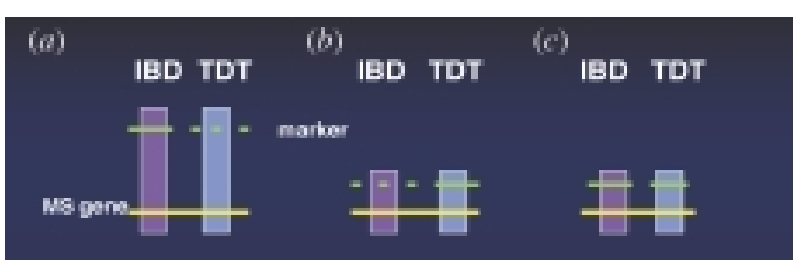

Figure 4. The relationship between linkage and association. Solid horizontal (green) bars indicate markers which are linked or associated with the putative MS susceptibility gene (yellow). Broken lines (green) indicate markers which are either not linked or associated. See the text for details. IBD, identical by descent; TDT, transmission disequilibrium testing. (a) Distant markers, linkage not associated, $(b)$ small sample, associated not linked and $(c)$ near marker, adequate sample, linked and associated.

parental allele has a 1:2 chance of being transmitted or not and the test provides evidence for an allelic association by demonstrating transmission disequilibrium in a sufficient sample size. TDT separately examines the movement of each allele which is present in the population and identifies that which is contributing to transmission disequilibrium in the sample. Not every family will have this marker but it follows that those which are in linkage disequilibrium with susceptibility genes will be well represented. One further factor limiting the extent to which the sample of family trios may not prove fully informative for a particular polymorphic locus is the presence of homozygotic parents; these are rejected in the analysis because one of the two identical copies must be transmitted and the other not. Segregation distortion arises when a particular allele confers a survival advantage and therefore appears to be disproportionately transmitted. In fact, this allele will be overrepresented in all offspring whether or not they have the disease trait in question. The issue can be resolved by studying affected and unaffected children in family quartets, but a new problem then arises which is offset by the affected family member approach and that is the difficulty in assigning the unaffected status to a young adult in a disease which may not manifest clinically until late in life, if at all, given the prevalence of pathologically verified but clinically silent disease in autopsy series. Because it is very unlikely that an investigator will chose to test for transmission disequilibrium at exactly the locus which encodes a disease susceptibility gene contributing to a complex trait, the dividend from family-based association methods also depends on the existence of linkage disequilibrium.

Linkage and association therefore provide different types of information. Each has its limitations and advantages. Assuming a reasonable sample size, any marker which is well distant from the true susceptibility locus will be linked but not associated. A marker which is close to the susceptibility locus will not be linked in sibling pairs if the sample size is inadequate (and, to date, most sibling pair studies are not) but it may be associated. Only the combination of a close marker tested in an adequate sample will demonstrate both linkage and association (figure 4).

\section{(c) Studies of candidate genes}

Population-based case-control studies have demonstrated an association between the class II major histocompatibility complex (MHC) alleles DR15 and DQ6 and their corresponding genotypes DRB1*1501, DRB5*0101 and DQA1*0102, DQB2*0602 (Olerup \& Hillert 1991) and the gene for tumour necrosis factor (TNF) $-\alpha$ is also usually associated (Coraddu et al. 1998) since this is encoded within the same linkage group. A specifically different association in seen in Mediterranean populations. For example, in Sardinians, MS is associated with DR4 (DRB1 *0405-DQA1 *0301, DQB1 *0302; Marrosu et al. 1992) although the DR15 association is seen among patients from all other parts of Italy in which adequate 


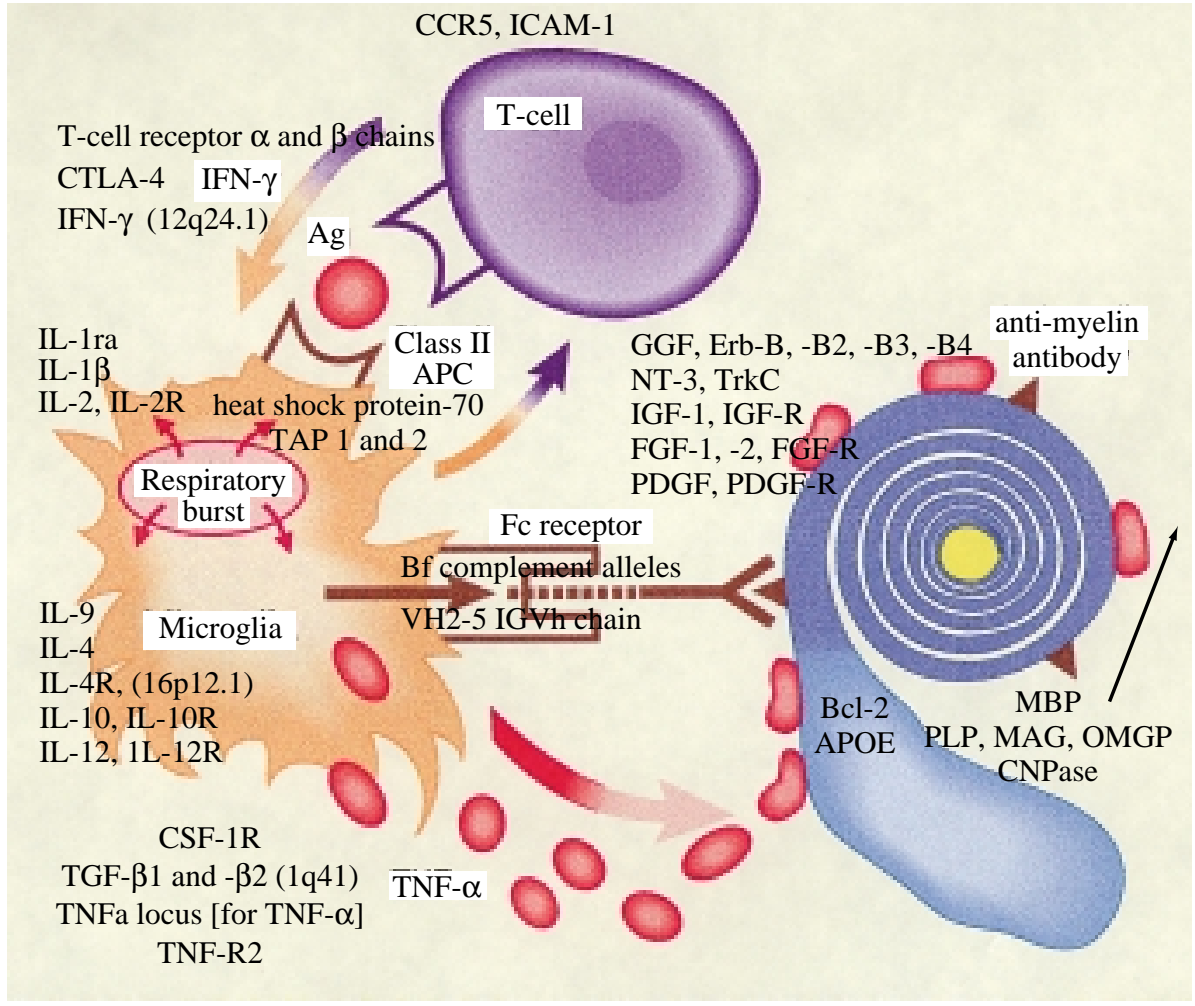

Figure 5. Positional and intelligent candidates tested for genetic susceptibility in MS based on a scheme for the pathogenesis. Most are uninformative but some show provisional evidence for linkage or an association. See the text for details and references. studies have been performed (La Mantia et al. 1990). In the Canary Islands, the disease is primarily associated with DR15 and DQ6 but a secondary association exists with DR4 (Coraddu et al. 1998). In Turkey, there is also an allelic association with both DR2 (DBR1*1501, DQA1*0102, DQB1 *0602) and DR4 (DRB1*04, DQA1*03, DQB1* 0302; Saruhan-Direskeneli et al. 1997).

Extensive searches using population- and family-based association and linkage studies over many years have only yielded additional putative candidate genes in the $\mathrm{VH} 2-5$ immunoglobulin heavy chain and the T-cell receptor (TCR) $\beta$-chain variable regions. Recent contributions to this aspect of the genetics of MS have tended to illustrate the difficulty which exists in confirming weak associations (VH2-5 (Ligers et al. 1997; Wansen et al. 1997) and TCR$\beta$ (Droogan et al. 1996; Wansen et al. 1997)). Studies of linkage or association with the genes encoding intercellular adhesion molecule (ICAM)-1 (Mycko et al. 1998), interleukin (IL)-1ra (De La Concha et al. 1997; Wansen et al. 1997), IL-1 $\beta$ and interferon (IFN) $\gamma$ (Wansen et al. 1997), IL-4 (Vanderbroeck et al. 1997) and 23 separate growth-factor genes and their receptors (Mertens et al. 1998) have all been negative. Except in the isolated population of Finns where there is both an association and linkage to the gene for myelin basic protein (Tienari et al. 1992, 1998), studies of structural genes of myelin have also been uninformative (Nellemann et al. 1995; Thompson et al. 1996; Price et al. 1997; Rodriguez et al. 1997; He et al. $1998 a$ ). Some investigators have explored new candidates. Using a variety of individuals (34 families, 147 cases and 95 controls), a Scandinavian group failed to implicate any one of several candidates encoding cytokines (IFN- $\gamma$, IL-2, IL-4, IL-10, transforming growth factor (TGF)- $\beta 1$ and $-\beta 2$ and IL-4R) or myelin proteins (proteolipid protein, myelin-associated glycoprotein, oligodendrocyte myelin glycoprotein and 2',3'-cyclic nucleotide- $3^{\prime}$-phos- phodiesterase, although IFN- $\gamma$ (12q24.1), IL-4R (16p12.1) and TGF- $\beta 2$ (lq41) could not be fully excluded (He et al. $1998 b)$ ). In France, efforts have been directed at growth factors which determine oligodendrocyte development and their receptors. Again, no evidence for linkage emerges but these may also be type 2 errors due to the small sample size (Reboul et al. 1999).

\section{(d) Systematic genome screens}

Four groups of investigators have undertaken a systematic search of the genome using affected family members in an attempt to locate additional susceptibility genes - usually using identity by descent analysis in sibling pairs. Genotyping was completed on cohorts of between 21 and 225 families, together involving in excess of 1000 individuals, for 257-443 microsatellite markers. These were chosen to have an average spacing of around $10 \mathrm{cM}$ giving enough power to identify regions encoding a major susceptibility gene and they are sufficiently polymorphic to make a high proportion of the available families fully informative. Although linkage analysis has revealed several new genomic regions which may encode genes conferring susceptibility to MS, some of these will be true and others false positives. Superficially, the results show a disappointing lack of overlap. In common with most other complex traits, no major susceptibility gene has been identified. The possible reasons are that no such gene exists, it has been missed by all three groups or heterogeneity has obscured the picture. The importance of the MHC is confirmed but, of the other new putative susceptibility loci, several are clearly unique to each screen and so may be false positives. The regions of interest emerging from the UK genome screen (Sawcer $e t$ al. 1996) are 1cen, 5 cen, 6p, 7p, 14q, 17q, 19q and Xp (see also Chataway et al. 1998) (figure 5). In the Canadian series they are 2p, 3p, 5p, 1lq and Xp (Ebers et al. 1996), 
while the US-French consortium has identified 6p, 7q, $11 p, 12 q$ and $19 q$ (Multiple Sclerosis Genetics Group 1996).

No statistically significant regions of interest were identified in the relatively small Finnish screen (Kuokkanen et al. 1997) although positive lod scores were achieved for 6p21 (MHC) and 5pl4-p12. Increasing the density of markers raised the lod scores in several other regions (4cen, 1ltel and 17q) whereas others (2q32 and 10 q21) were unchanged. When all 21 families were typed across the regions of interest, the highest lod score (2.8) was at $17 \mathrm{q} 22-\mathrm{q} 24$, as in the previously reported UK screen.

The suggestion of meta-analysis has been repeatedly made. Areas shared between the MS screens can be detected by eye and have been listed in various reviews but integrated analysis of the raw genotypes as a means of ironing out similarities and discrepancies between the screens has not yet materialized. An alternative has been to pool information from screens in specifically different complex traits searching for overlapping regions which might, for example, encode autoimmune genes. Becker et al. (1998) studied 23 full genome screens and concluded that around $65 \%$ of the positive linkages map nonrandomly to 18 distinct clusters; some are shared by more than one human disorder and are syntenic to loci implicated in experimental models of autoimmunity. Merriman and colleagues have implicated a candidate locus on 18q12-21 using transmission disequilibrium in 2383 simplex families with type 1 diabetes, 936 with MS and 309 with rheumatoid arthritis $\left(n=3649 ; p=5 \times 10^{-8}\right.$ for association using TDT adjusted for linkage, $\mathrm{T}_{\mathrm{sp}}$ ) and suggested that this encodes a gene regulating tolerance and immune homeostasis (A. Merriman and 24 others, unpublished data).

\section{(e) Positional candidates}

The most logical way to proceed from the identification of regions of interest in full genome screens is to concentrate on new candidates located under the linkage peaks. This presupposes that the provisional identification of these hits is valid and it makes sense to remain alert to the possibility of new intelligent candidates encoded outside these regions, based on evolving concepts of the pathogenesis (figure 6).

In a study from Scandinavia (Xu et al. 1999), 15 markers from regions of interest identified in one or more full genome screens were assessed using affected relative pairs (from 46 multiplex families, 28 trios, 190 cases and 148 unrelated controls). Depending on the method of analysis, positive results were obtained for 5p15, 6p21, 7 ptr15 and 12q23; the latter two also showed transmission disequilibrium in families and an association in the casecontrol series. The remaining markers (2p23, 5p, 5q, 6q22-25, 7q, 1lq21-23, 12q24-qtr, 13q33-34, 16p12, $18 \mathrm{p} 11$ and $\mathrm{Xp21}$ ) did not identify regions of interest in the Scandinavian series. An Italian group used 67 markers covering regions of interest from the three full genome screens and candidates (HLA-DRB1, cytotoxic lymphocyte-associated antigen (CTLA)-4, IL-9, colonystimulating factor (CSF)-1R, apoliprotein (APO)-E, Bcl-2 and TNF-R2) in 69 multiplex families (28 from Sardinia and 41 from mainland Italy). Some positive scores were obtained particularly for the region at $5 \mathrm{q} 14$ in the Sardinian subset. Three (2p11.2, 7p15.2 and 17q12) also showed transmission disequilibrium (D'Alfonso et al. 1999). Oturai et al. (1999) confirmed linkage but no association of a pericentromeric region on chromosome 5 and of 6 p21 to susceptibility in MS in sibling pairs from Scandinavia.

Chromosome 5 has now featured in all the family studies but the region is broad and it may be difficult to assess in more detail.

Chataway et al. (1999a) used a multiplexed oligoligation assay to test single nucleotide polymorphisms from within the C6 and C7 complement genes encoded under the peak on the pericentromeric region of chromosome 5 in both case-control comparisons and using transmission disequilibrium testing, but failed to implicate any one allele of these candidates in susceptibility to MS.

In view of its potential role in retrovirus pathogenesis, Bennetts et al. (1997) compared the frequency of chemokine receptor CGR5 mutations in cases and controls from Australia but found no difference. Chataway et al. (1999b) also failed to show transmission disequilibrium for alleles encoded at this locus but the study confirmed that the delta CCR5 mutation forms part of the same haplotype made up from neighbouring loci. This provides clear evidence for a genetic founder effect and establishes that linkage disequilibrium does exist within the human genome among outbred Europeans.

Chataway et al. (1999c) also examined a selection of candidates located under peaks from one or more of the four full genome screens using TDT in 744 family trios. These included the APO-C loci and the gene for myelin associated glycoprotein on chromosome 19, the genes for CNPase, myeloperoxidase, neurofibromin and APO-H at various locations on chromosome 17, tissue inhibitor of metalloproteinase 3 and neurofibromatosis 2 on chromosome 22, the gene for microtubule-associated protein $1 \mathrm{~B}$ on chromosome 5 and APO-B on chromosome 2. Out of these, only the 192bp allele of myeloperoxidase showed excess transmission.

In a population association study, Barcellos et al. (1997) showed an increase in the 19q13.2 haplotype in Caucasian patients with MS from California; they confirmed the HLA haplotype association (6p21.3) in the same population but not the association with myelin basic protein (18q23).

\section{(f) Heterogeneity}

These genetic analyses are predicated on the assumption that MS is one disease. The issue of heterogeneity is arguably the most important unexplored aspect of the genetics of MS. Given the variable clinical features and course, the diversity of pathological and imaging changes and the hints of genetic differences within and between regions, it is to be expected that examples of specifically different diseases and more than one mechanistic route to demyelination are masquerading under the rubric of clinically definite MS.

Mutations of mitochondrial DNA are responsible for a MS-like illness characterized by disproportionate involvement of the anterior visual pathway (Harding et al. 1992; Riordan Eva et al. 1995), although mitochondrial genes do not generally contribute to susceptibility in MS 

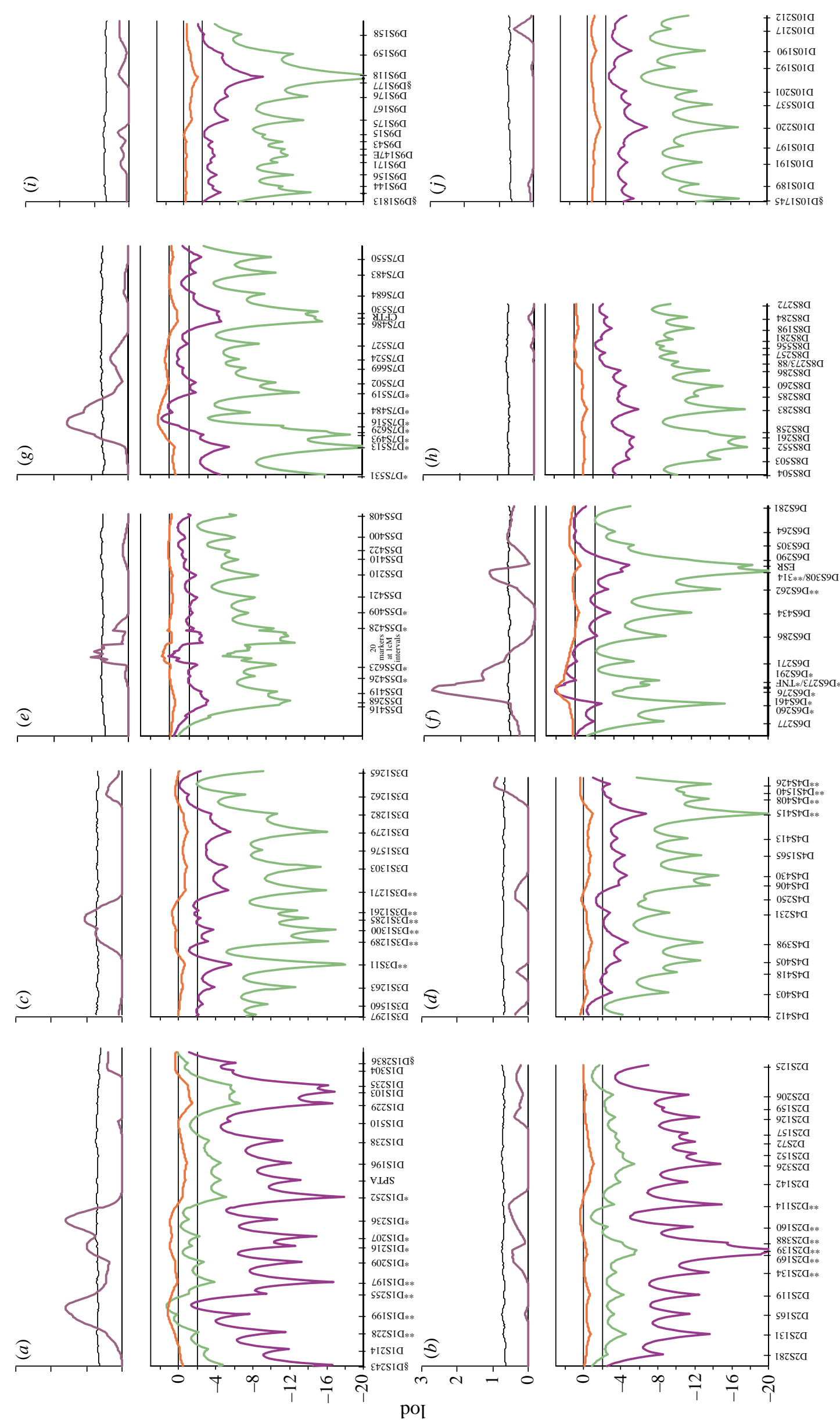

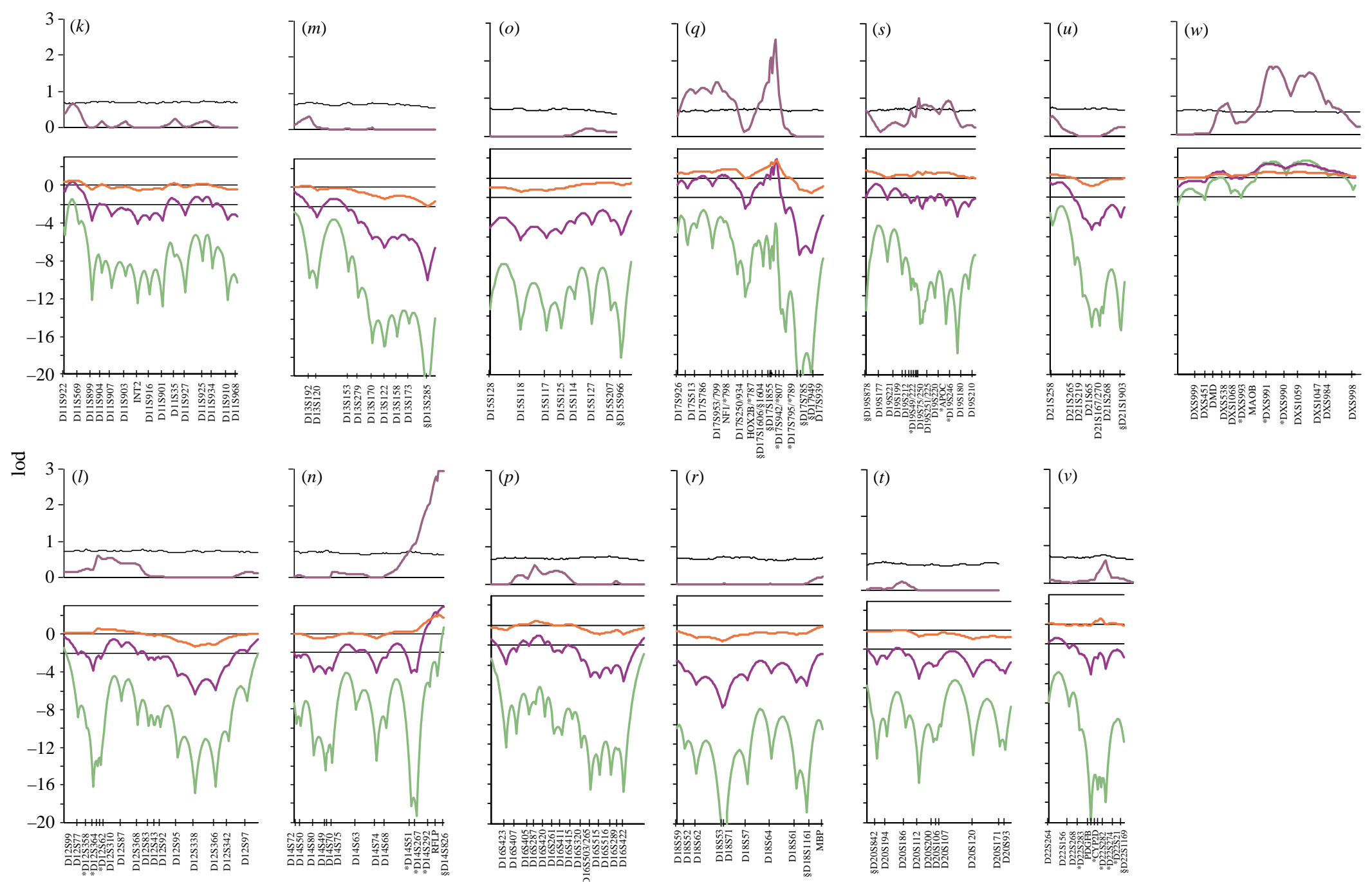

Figure 6. Maximum lod scores and exclusion maps for the human genome in MS (from Chataway et al. 1998). The horizontal axis is proportional to the length of each chromosome and maps the individual markers. The upper vertical axis shows the maximum lod score for each marker in a multipoint analysis. Exclusion maps are shown under a model of no dominance for genes exerting a $\lambda_{\mathrm{s}}$ of 5 (green), 2 (blue purple) and 1.2 (red). (a j) Chromosomes 1-10, $(k v)$ chromosomes 11-22 and (w) chromosome X. 
(Kellar Wood et al. 1994; Kalman et al. 1995; Nishimura et al. 1995).

Based on the estimation of very long chain fatty acids in male-male pairs, there were no examples of adrenoleucodystrophy in the UK cohort of siblings (Sawcer et al. 1996). Chataway et al. (1999d) returned to the issue of phenocopies and excluded examples of spinocerebellar degeneration (SCA1, 2, 3, 6 and 7) from this sample. However, there was excess transmission of the 22 trinucleotide repeat allele of SCA2 encoded on chromosome 12. One explanation is that the presence of a particular allele encoded at a locus where trinucleotide expansions determine premature degeneration of a defined axonal pathway may promote axonal pathology and direct the disease process towards a particular system in MS.

Conditioning the UK genome screen for DR15 (or an extended DR15-linked haplotype also encoding alleles of TNF and the DQ locus) shows that the regions of interest on $1 p, 17 p, 17 q$ and $\mathrm{X}$ cluster in families which are identical by state for DR15, whereas the non-sharing group is associated with 1cen, 3p, 5cen, 7p, 14q and 22q (Coraddu et al. 1999). In addition, new regions of interest are found at $5 q$ and 13p (DR15 sharing) and 16p and 20p (DR15 nonsharing). A major part of future studies in the genetics of MS will be to resolve the question of disease heterogeneity.

\section{(g) Future strategies}

The issue for the future is how best to pick the regions of interest and identify the responsible genes encoded therein reliably. A number of tactics can be proposed for resolving the status of these regions of interest. The obvious strategy is to increase the number of families and the density of markers at specific loci and to complete the search in relatively unexplored regions. These approaches provide rather little new guidance and they are limited by the small return on extraction of new information (Chataway et al. 1998). More importantly, once the density of markers gets much above $5 \mathrm{cM}$, typing errors and inconsistencies in the map order begin to corrupt the results and multipoint maps degrade (Feakes et al. 1999). Based on current strategies for complex traits and the available results, it might seem logical to focus on family-based association studies. Here, the problem is that, for a systematic search, the extent of linkage disequilibrium in outbred populations demands a very dense set of markers and, therefore, a prodigious amount of genotyping. That restriction may not apply to genetic isolates who happen to have a high prevalence of a complex trait such as MS and much more extensive linkage disequilibrium. Thus, one strategy is first to screen the genome with linkage to establish regions of interest and then to refine the map with TDT. However, laboratory methods are beginning to be deployed which make whole genome linkage disequilibrium screening feasible. The necessary microsatellite and single nucleotide polymorphism markers are available and the number of trios needed for a definitive study is more manageable than that required for linkage using affected sibling pairs. A preferred strategy is therefore first to screen cases and controls using pooled DNA (that is, two samples each made up from several hundred individuals) so as to identify those markers which show a difference in frequency. Pooling can reliably detect a $5 \%$ difference in allele frequency. Some results will be false positives and it is therefore necessary as a second step to retype the individuals across the emerging regions of interest, using TDT with a suitably dense set of markers.

The contribution to susceptibility made by the regions which have provisionally been identified only accounts for a proportion of the increased risk of MS implicated by family studies. Clearly there is more to be learned concerning susceptibility to MS and the interplay between genes and environmental factors. Sustained effort is worthwhile since the application of this knowledge for improved understanding of the pathogenesis in counselling and in designing novel treatments is potentially considerable.

\section{REFERENCES}

Andersen, O., Lygner, P.-E., Berstrom, T., Andersson, M. \& Vahlne, A. 1993 Viral infections trigger multiple sclerosis relapses: a prospective sero-epidemiological study. F. Neurol. 240, 417-422.

Barcellos, L. F., Thomson, G., Carrington, M., Schafer, J., Begovich, A. B., Lin, P., Xu, X. H., Min, B. Q., Marte, D. \& Klitz, W. B. 1997 Chromosome 19 single-locus and multilocus haplotype associations with multiple sclerosis. Evidence of a new susceptibility locus in Caucasian and Chinese patients. $\mathcal{F}$. Am. Med. Assoc. 278, 1256-1261.

Becker, K. G., Simon, R. M. \& Bailey-Wilson, J. E. 1998 Clustering of non-human histocompatibility complex susceptibility candidate loci in human autoimmune diseases. Proc. Natl Acad. Sci. USA 95, 9979-9984.

Benedikz, J. G., Magnusson, H. \& Gudmundsson, G. 1994 Multiple sclerosis in Iceland, with observations on the alleged epidemic in the Faroe Islands. Ann. Neurol. 36 (Suppl. 2), S175-S179.

Bennetts, B. H., Teutsch, S. M., Buhler, M. M., Heard, R. N. \& Stewart, G. J. 1997 The CCR5 deletion mutation fails to protect against multiple sclerosis. Hum. Immunol. 58, 52-59.

Bulman, D. E., Sadovnick, A. D., Cripps, J. \& Ebers, G. C. 1991 Age of onset in siblings concordant for multiple sclerosis. Brain 114, 937-950.

Carton, H., Vlietinck, R., Debruyne, J., De Keyser, J., D'Hooghe, M.-B., Loos, R., Medaer, R., Truyen, L., Yee, I. M. L. \& Sadovnick, D. 1997 Recurrence risks of multiple sclerosis in relatives of patients in Flanders, Belgium. f. Neurol. Neurosurg. Psychiatr. 62, 329-333.

Chataway, J., Feakes, R., Coraddu, F., Gray, J., Deans, J., Fraser, M., Robertson, N. P., Broadley, S., Jones, H., Clayton, D., Goodfellow, P. N., Sawcer, S. \& Compston, D. A. S. 1998 The genetics of multiple sclerosis: principles, background and updated results of the United Kingdom systematic genome screen. Brain 121, 1869-1887.

Chataway, J., Sawcer, S., Sherma, D., Hobart, M., Fernie, B., Coraddu, F., Fleakes, R., Broadley, S., Gray, J., Jones, H. B., Clayton, D., Goodfellow, P. N. \& Compston, D. A. S. $1999 a$ No evidence for association of multiple sclerosis with the complement factors C6 and C7. 7. Neuroimmunol. (In the press.)

Chataway, J., Sawcer, S., Feakes, R., Coraddu, F., Broadley, S. D., Gray, J. \& Compston, D. A. S. 1999 b More evidence that founder effects exist in the European population. Eur. F. Hum. Genet. (In the press.)

Chataway, J., Sawcer, S., Feakes, R., Coraddu, F., Broadley, S. D., Jones, H. B., Glayton, D., Gray, J., Goodfellow, P. N. \& Compston, D. A. S. 1999c A screen of candidates from peaks of linkage: evidence for the involvement of myeloperoxidase in multiple sclerosis. F. Neuroimmunol. (In the press.) 
Chataway, J., Sawcer, S., Coraddu, F., Feakes, R., Broadley, S., Jones, H. N., Glayton, D., Gray, J., Goodfellow, P. N. \& Compston, D. A. S. 1999d Allelic variants of the spinocerebellar ataxia genes contribute to multiple sclerosis susceptibility. Neurogenetics. (In the press.)

Compston, D. A. S. (ed.), Ebers, G. C., Lassmann, H., McDonald, W. I., Matthews, W. B. \& Wekerle, H. 1998 McAlpine's multiple sclerosis. London: Churchill Livingstone.

Confavreux, C., Hutchinson, M., Marie-Hours, M., Coutinovis Tourniaire, P. \& Moreau, T. 1998 Rate of pregnancy-related relapse in multiple sclerosis. $\mathcal{N}$. Engl. F. Med. 339, 285-291.

Coraddu, F., Reyes-Yanez, M. P., Aladro, Y., Parra, A., Gray, J., Smith, S., Taylor, C. \& Compston, D. A. S. 1998 HLA associations with multiple sclerosis in the Canary Islands. $\mathcal{F}$. Neuroimmunol. 87, 130-135.

Coraddu, F., Sawcer, S., Feakes, R., Chataway, J., Broadley, S., Jones, H. B., Clayton, D., Gary, J., Smith, S., Taylor, C., Goodfellow, P. N. \& Compston, D. A. S. 1999 HLA conditioning in a multiple sclerosis genome screen. Neurogenetics 2, 24-33.

D’Alfonso, S. (and 19 others) 1999 A linkage analysis of multiple sclerosis with candidate region markers in Sardinian and continental Italian families.

Dean, G. 1967 Annual incidence, prevalence and mortality of MS in White South African-born and in White immigrants to South Africa. Br. Med. 7. 2, 724-730.

De La Concha, E. G., Arroyo, R., Crusius, J. B., Campillo, J. A., Martin, C., Varela de Seyas, E., Pena, A. S., Claveria, L. E. \& Fernandez Arquero, M. 1997 Combined effect of HLA-DRB1 ${ }^{*} 1501$ and interleukin-1 receptor antagonist gene allele 2 in susceptibility to relapsing/remitting multiple sclerosis. F. Neuroimmunol. 80, 172-178.

Droogan, A. G., Kirk, C. W., Hawkins, S. A., McMillan, S. A., Nevin, N. C. \& Graham, C. A. 1996 T cell receptor alpha, beta, gamma and delta chain microsatellites show no association with multiple sclerosis. Neurology 47, 1049-1053.

Ebers, G. C., Sadovnick, A. D. \& Risch, N. J. 1995 A genetic basis for familial aggregation in multiple sclerosis. Nature 377, 150-151.

Ebers, G. C. (and 29 others) 1996 A full genome search in multiple sclerosis. Nature Genet. 13, 472-476.

Elian, M. \& Dean, G. 1987 Multiple sclerosis among United Kingdom born children of immigrants from the West Indies. 7. Neurol. Neurosurg. Psychiatr. 50, 327-332.

Feakes, R., Chataway, J., Sawcer, S. et al. 1999 Exploring the dense mapping of potential linkage in complex disease: an example in multiple sclerosis. Genet. Epidemiol. 17, 51-63.

French Research Group on Multiple Sclerosis 1992 Multiple sclerosis in 54 twinships: concordance rate is independent of zygosity. A. Neurol. 32, 724-727.

Granieri, E. \& Casseta, I. 1997 Common childhood and adolescent infections and multiple sclerosis. Neurology 49 (Suppl. 2), S42-S54.

Harding, A. E., Sweeney, M. G., Brockington, M., Muller, D. H., Mumford, C. J., Kellar-Wood, H., Compston, D. A. S. \& McDonald, W. I. 1992 Occurrence of a multiple sclerosislike illness in women who have a Leber's hereditary optic neuropathy mitochondrial DNA mutation. Brain 115, 989-989.

He, B., Yang, B., Lundahl, J., Fredrikson, S. \& Hellert, J. $1998 a$ The myelin basic protein gene in multiple sclerosis: identification of discrete alleles of a $1.3 \mathrm{~kb}$ tetranucleotide repeat sequence. Acta Neurol. Scand. 97, 46-51.

He, B., Xu, C., Yang, B., Landtblom, A.-M., Fredrikson, S. \& Hellert, J. $1998 b$ Linkage and association analysis of genes encoding cytokines and myelin proteins in multiple sclerosis. 7. Neuroimmunol. 86, 13-19.

Kahana, E., Zilber, N., Abramson, J. H., Biton, Y., Leibowitz, Y. \& Abramsky, O. 1994 Multiple sclerosis: genetic versus envir- onmental aetiology: epidemiology in Israel updated. F. Neurol. 241, 341-346.

Kalman, B., Lublin, F. D. \& Alder, H. 1995 Mitochondrial DNA mutations in multiple sclerosis. Multiple Sclerosis 1, 32-36.

Kellar Wood, H., Robertson, N., Govan, G. G., Harding, A. E. \& Compston, D. A. S. 1994 Leber's hereditary optic neuropathy mitochondrial DNA mutations in multiple sclerosis. Ann. Neurol. 36, 109-112.

Kuokkanen, S. (and 11 others) 1997 Genomewide scan of multiple sclerosis in Finnish multiplex families. Am. 7. Hum. Genet. 61, 1379-1387.

Kurtzke, J. F. 1975 A reassessment of the distribution of multiple sclerosis. Acta Neurol. Scand. 51, 110-157.

Kurtzke, J. F. 1993 Epidemiologic evidence for multiple sclerosis as an infection. Clin. Microbiol. Rev. 6, 382-427.

Kurtzke, J. F., Gudmundsson, K. R. \& Bergmann, S. 1982 Multiple sclerosis in Iceland. 1. Evidence of a post-war epidemic. Neurology 32, 143-150.

La Mantia, L., Illeni, M. T., Milanese, C., Salmaggi, A., Eoli, M., Pellegris, G. \& Nespolo, A. 1990 HLA and multiple sclerosis in Italy: a review of the literature. F. Neurol. 237, $441-444$.

Ligers, A., He, B., Fogdell-Hahn, A., Olerup, O. \& Hilleret, J. 1997 No linkage or association of a VNTR marker in the junction region of the immunoglobulin heavy chain genes in multiple sclerosis. Eur. 7. Immunogenet. 24, 259-264.

Marrosu, M. G., Muntoni, F., Murru, M. R., Costa, G., Pischedda, M. P., Piratsu, M., Sotgin, S., Rosati, G. \& Cinchetti, C. 1992 HLA-DQB1 genotype in Sardinian multiple sclerosis: evidence for a key role of DQB1.0201 and DQB1.0302 alleles. Neurology 42, 883-886.

Martyn, C. N., Cruddas, M. \& Compston, D. A. S. 1993 Symptomatic Epstein-Barr virus infection and multiple sclerosis. 7. Neurol. Neurosurg. Psychiatr. 56, 167-168.

Mertens, C. (and 14 others) 1998 A systematic study of oligodendrocyte growth factors as candidates for genetic susceptibility to MS. Neurology 51, 748-753.

Multiple Sclerosis Genetics Group 1996 A complete genomic screen for multiple sclerosis underscores a role for the major histocompatibility complex. Nature Genet. 13, 469-471.

Mumford, C. J., Wood, N. W., Kellar-Wood, H. F., Thorpe, J., Miller, D. \& Compston, D. A. S. 1994 The British Isles survey of multiple sclerosis in twins. Neurology 44, 11-15.

Mycko, M. P., Kwinkowoski, M., Tronczynska, E., Szymanska, B. \& Selmaj, K. W. 1998 Multiple sclerosis: increased frequency of the ICAM-1 exon 6 gene point mutation genetic type K469. Ann. Neurol. 44, 70-75.

Nellemann, L. J., Frederiksen, J. \& Morling, N. 1995 PCR typing of two short tandem repeat (STR) structures upstream of the human myelin basic protein (MBP) gene; genetic susceptibility in multiple sclerosis and monosymptomatic idiopathic optic neuritis in Danes. Multiple Sclerosis 1, 186-189.

Nishimura, M., Obayashi, H., Ohta, M., Uchiyama, T., Hao, Q. \& Saida, T. 1995 No association of the 11778 mitochondrial mutation and multiple sclerosis in Japan. Neurology 45, 1333-1334.

Olerup, O. \& Hillert, J. 1991 HLA class II-associated genetic susceptibility in multiple sclerosis: a critical evaluation. Tiss. Antigens 38, 1-15.

Operskalski, E. A., Visscher, B. R., Malgren, R. M. \& Detels, R. 1989 A case control study of multiple sclerosis. Neurology 39, 825-829.

Oturai, A. (and 12 others) 1999 Linkage and association analysis of susceptibility regions on chromosomes 5 and 6 in 106 Scandinavian sib pair families with multiple sclerosis. Ann. Neurol. (In the press.)

Price, S. E., Sharpe, G., Boots, A., Poutsma, A., Mason, G., James, J., Hinks, L. \& Thompson, R. J. 1997 Role of myelin 
basic protein and proteolipid protein genes in multiple sclerosis: single strand conformation polymorphism analysis of the human sequences. Neuropathol. Appl. Neurobiol. 23, 457-467.

Reboul, J. (and 15 others) 1999 Cytokines in genetic susceptibility to multiple sclerosis: a candidate gene approach. (In the press.)

Riordan Eva, P., Sanders, M. D., Govan, G. G., Sweeney, M. G., Da Costa, J. \& Harding, A. E. 1995 The clinical features of Leber's hereditary optic neuropathy defined by the presence of pathogenic mitochondrial DNA mutation. Brain 118, 319-337.

Risch, N. 1990 Linkage strategies for genetically complex traits. Am. F. Hum. Genet. 46, 222-253.

Robertson, N. P., Clayton, D., Fraser, M., Deans, J. \& Compston, D. A. S. $1996 a$ Clinical concordance in sibling pairs with multiple sclerosis. Neurology 47, 347-352.

Robertson, N. P., Fraser, M., Deans, J., Clayton, D. \& Compston, D. A. S. $1996 b$ Age adjusted recurrence risks for relatives of patients with multiple sclerosis. Brain 119, 449-455.

Robertson, N. P., O'Riordan, J. I., Chataway, J., Kingsley, D. P. E., Miller, D. H., Clayton, D. \& Compston, D. A. S. 1997 Clinical characteristics and offspring recurrence rates of conjugal multiple sclerosis. Lancet 349, 1587-1590.

Rodriguez, D., Della Gaspera, B., Zalc, B., Hauw, J. J., Fontaine, B., Edan, G., Clanet, M., Dautigny, A. \& Pham Dinh, D. 1997 Identification of a Val 145 Ile substitution in the human myelin oligodendrocyte glycoprotein: lack of association with multiple sclerosis. Multiple Sclerosis 3, 377-382.

Runmarker, B. \& Andersen, O. 1995 Pregnancy is associated with a lower risk of onset and a better prognosis in multiple sclerosis. Brain 118, 253-261.

Sadovnick, A. D., Baird, P. A. \& Ward, R. H. 1988 Multiple sclerosis; updated risks for relatives. Am. 7. Med. Genet. 29, 533-541.

Sadovnick, A. D. (and 18 others) 1993 A population-based study of multiple sclerosis in twins: update. Ann. Neurol. 33, 281-285.

Sadovnick, A. D., Ebers, G. C., Dyment, D. A., Risch, N. \& the Canadian Collaborative Study Group 1996 Evidence for genetic basis of multiple sclerosis. Lancet 347, 1728-1730.

Saruhan-Direskeneli, G., Esin, S., Baykan-Kurt, B., Ornek, I., Vaughan, R. \& Eraksoy, M. 1997 HLA-DR and -DQ associations with multiple sclerosis in Turkey. Hum. Immunol. 55, 59-65.

Sawcer, S., Jones, H. B., Feakes, R., Gray, J., Smaldon, N., Chataway, J., Robertson, N., Clayton, D., Goodfellow, P. N. \& Compston, D. A. S. 1996 A genome screen in multiple sclerosis reveals susceptibility loci on chromosome $6 \mathrm{p} 21$ and 17q22. Nature Genet. 13, 464-468.

Sibley, W. A., Bamford, C. R. \& Clark, K. 1985 Clinical viral infections and multiple sclerosis. Lancet $\mathbf{i}, 1313-1315$.

Sibley, W. A., Bamford, C. R., Clark, K., Smith, M. S. \& Laguna, J. F. 1991 A prospective study of physical trauma and multiple sclerosis. 7. Neurol. Neurosurg. Psychiatr. 54, 584-589.

Siva, A., Radhakrishnan, K., Kurland, L. T., O’Brien, P. C., Swanson, J. W. \& Rodriquez, M. 1993 Trauma and multiple sclerosis: a population based cohort study from Olmsted County, Minnesota. Neurology 43, 1878-1882.

Thompson, R. J., Mason, C. R., Douglas, A. J., Hinks, L. J., Dwarswaard, A. \& Price, S. E. 1996 Analysis of polymorphisms of the $2^{\prime} 3^{\prime}$-cyclic nucleotide- $3^{\prime}$-phosphodiesterase gene in patients with multiple sclerosis. Multiple Sclerosis 2, 215-221.

Tienari, P., Wikstrom, J., Sajantila, A., Palo, J. \& Peltonen, L. 1992 Genetic susceptibility to multiple sclerosis linked to myelin basic protein gene. Lancet 340, 987-991.

Tienari, P., Kuokkanen, S., Pastinen, T., Wikstrom, J., Sajantila, M., Sandberg Wolheim, M., Palo, J. \& Peltonen, L. 1998 Golli-MBP gene in multiple sclerosis. 7. Neuroimmunol. 81, 158-167.

Vanderbroeck, K., Martino, G., Marrosu, M., Consiglio, A., Zaffaroni, M., Vaccargiu, S., Franciotta, D., Ruggeri, M., Comi, G. \& Grimaldi, L. M. 1997 Occurrence and clinical relevance of an interleukin- 4 gene polymorphism in patients with multiple sclerosis. 7. Neuroimmunol. 76, 189-192.

Wansen, K., Pastinen, T., Kuokkanen, S., Wokstrom, J., Wikstrom, J., Palo, J., Peltonen, L. \& Tienari, P. J. 1997 Immune system genes in multiple sclerosis: genetic association and linkage analyses on TCR- $\beta$, IgH, IFN- $\gamma$ and IL-12ra/IL$1 \beta$ loci. F. Neurol. 79, 29-36.

Xu, C., Dai, Y., Fredrikson, \& Hillert, J. 1999 Association and linkage analysis of candidate chromosomal regions in multiple sclerosis: indication of disease genes in 12q23. Eur. 7. Hum. Genet. 7, 110-116. 